\title{
Synthesis of C-rich dust in CO nova outbursts
}

\author{
Jordi José ${ }^{1,2}$, Ghina M. Halabi ${ }^{3}$, and Mounib F. El Eid ${ }^{4}$ \\ 1 Departament de Física, EUETIB, Universitat Politècnica de Catalunya, c/Comte d'Urgell 187, 08036 Barcelona, Spain \\ e-mail: jordi.jose@upc.edu \\ 2 Institut d'Estudis Espacials de Catalunya, c/Gran Capità 2-4, Ed. Nexus-201, 08034 Barcelona, Spain \\ 3 Institute of Astronomy, University of Cambridge, Madingley Road, Cambridge CB3 OHA, UK \\ ${ }^{4}$ Department of Physics, American University of Beirut, Bliss St. 11-0236, 11072020 Beirut, Lebanon
}

Received 11 May 2016 / Accepted 15 June 2016

\begin{abstract}
Context. Classical novae are thermonuclear explosions that take place in the envelopes of accreting white dwarfs in stellar binary systems. The material transferred onto the white dwarf piles up under degenerate conditions, driving a thermonuclear runaway. In these outbursts, about $10^{-7}-10^{-3} M_{\odot}$, enriched in $\mathrm{CNO}$ and sometimes other intermediate-mass elements (e.g., $\mathrm{Ne}, \mathrm{Na}, \mathrm{Mg}$, or $\mathrm{Al}$ for ONe novae) are ejected into the interstellar medium. The large concentrations of metals spectroscopically inferred in the nova ejecta reveal that the solar-like material transferred from the secondary mixes with the outermost layers of the underlying white dwarf.

Aims. Most theoretical models of nova outbursts reported to date yield, on average, outflows characterized by $\mathrm{O}>\mathrm{C}$, from which, in principle, only oxidized condensates (e.g., O-rich grains) would be expected.

Methods. To specifically address whether CO novae can actually produce C-rich dust, six different hydrodynamic nova models have been evolved, from accretion to the expansion and ejection stages, with different choices for the composition of the substrate with which the solar-like accreted material mixes. Updated chemical profiles inside the H-exhausted core have been used, based on stellar evolution calculations for a progenitor of $8 M_{\odot}$ through $\mathrm{H}$ - and He-burning phases.

Results. We show that these profiles lead to C-rich ejecta after the nova outburst. This extends the possible contribution of novae to the inventory of presolar grains identified in meteorites, particularly in a number of carbonaceous phases (i.e., nanodiamonds, silicon carbides, and graphites).
\end{abstract}

Key words. novae, cataclysmic variables - nuclear reactions, nucleosynthesis, abundances - white dwarfs

\section{Introduction}

Classical novae are prolific dust producers. Infrared and ultraviolet observations have unambiguously revealed a number of dust forming episodes in the ejected shells accompanying nova outbursts (Evans 1990; Evans \& Rawlings 2008; Gehrz et al. 1998; Gehrz 2002, 2008; Shore et al. 1994). As first suggested by Stratton \& Manning (1939) on the occasion of nova DQ Her, dust formation is revealed by a rise in infrared emission that occurs simultaneously with a decline in the optical lightcurve, several months after peak luminosity.

Such dust forming episodes are driven by the fast decline in temperature and density in the nova outflows, which allows the plasma to recombine and subsequently form molecules. Grain formation (i.e., dust) is a complex process dictated by the local thermodynamical and physicochemical conditions of the environment, requiring moderately low temperatures (below $1500-2000 \mathrm{~K})$ and particle densities $\left(\geq 10^{8} \mathrm{~cm}^{-3}\right)$. Roughly speaking, it can be qualitatively described as a two-stage process: grain nucleation and growth to macroscopic size (Sedlmayr 1994; Lodders 2003; Andersen 2011). In the former, a handful of molecules assemble into small groups or clusters, which by effect of a suite of chemical processes grow to a critical size. The carbon monoxide (CO) molecule plays an important role in this process. While most heteronuclear, diatomic molecules have dissociation energies around $3-5 \mathrm{eV}$, the $\mathrm{CO}$ molecule is characterized by a striking bond energy of $11.2 \mathrm{eV}$. Therefore, it is a very stable molecule, and can only be dissociated by high-energy photons. In the absence of intense radiation fields, condensation in an O-rich plasma (i.e., $\mathrm{O}>\mathrm{C}$ ) results in nearly all carbon atoms being locked in the form of the very stable $\mathrm{CO}$ molecules. As a result, formation of C-rich grains, such as silicon carbides $(\mathrm{SiC})$, graphites, or nanodiamonds, is unlikely. Conversely, in a $\mathrm{C}$-rich environment $(\mathrm{C}>\mathrm{O})$, all oxygen gets trapped in $\mathrm{CO}$ molecules and, in principle, oxidized compounds (e.g., corundum, spinel, hibonite, enstatite) cannot form.

Infrared measurements of a number of novae have actually revealed the presence of C-rich dust (Aql 1995, V838 Her 1991, PW Vul 1984), SiC (Aql 1982, V842 Cen 1986), hydrocarbons (V842 Cen 1986, V705 Cas 1993), or $\mathrm{SiO}_{2}$ (V1370 Aql 1982, V705 Cas 1993) in the ejecta. Moreover, sequential formation of all these types of dust has also been reported for some remarkable novae, such as V842 Cen 1986 (Gehrz et al. 1990) and QV Vul 1987 (Gehrz et al. 1998). In fact, it has been suggested that novae may have contributed to the different presolar grain populations isolated from meteorites ${ }^{1}$ (Clayton \& Hoyle 1976; Amari et al. 2001; Amari 2002; Jose et al. 2004; Haenecour et al. 2016). Surprisingly, most theoretical models of nova outbursts (even those that rely on a CO-rich white dwarf as the underlying compact object that hosts the outburst) yield, on average, outflows characterized by $\mathrm{O}>\mathrm{C}$, from which only oxidized

\footnotetext{
1 See also Pepin et al. (2011) for the possible nova origin of anomalous interplanetary dust particles collected from comets Grigg-Skjellerup and Tempel-Tuttle.
} 
condensates would be expected ${ }^{2}$. Equilibrium condensation sequences have suggested, however, that the presence of large amounts of intermediate-mass elements, such as $\mathrm{Al}, \mathrm{Ca}, \mathrm{Mg}$, or $\mathrm{Si}$, may dramatically alter the condensation process, allowing the formation of C-rich dust even in a slightly O-rich environment (José et al. 2004). Moreover, it is expected that dust condensation in a nova environment proceeds kinetically rather than at equilibrium because of the strong radiation emitted by the underlying white dwarf (see, e.g., Shore \& Gehrz 2004). Both effects reduce the significance of the $\mathrm{CO}$ molecule in the process of dust formation. But whether the nova ejecta are actually $\mathrm{C}$ - or O-rich has not been systematically addressed in the framework of hydrodynamic simulations. Indeed, nova models have traditionally assumed that the composition of the underlying white dwarf star that hosts the outbursts has similar mass fractions of ${ }^{12} \mathrm{C}$ and ${ }^{16} \mathrm{O}$ when low-mass, CO-rich white dwarfs are considered (see, however, Kovetz \& Prialnik 1997, for a study of the effect of the white dwarf composition on the basis of pure-C, pure-O, and standard $\mathrm{C} / \mathrm{O}=1$ white dwarf models).

In this paper, a series of nova models are presented, with state-of-the-art chemical profiles based on new evolutionary sequences of intermediate-mass stars that lead to the formation of white dwarfs with outer layers characterized by $\mathrm{C}>\mathrm{O}$. An outline of the method of computation and input physics used in this paper is given in Sect. 2. The impact of the abundance profiles of the underlying white dwarf on the nucleosynthesis accompanying classical nova outbursts is presented in Sect. 3. Finally, the most relevant conclusions of this paper are summarized in Sect. 4.

\section{Input physics and initial setup}

The nova models reported in this paper have been computed with the 1D, implicit, Lagrangian hydrodynamic code SHIVA, extensively used in the modeling of stellar explosions (e.g., classical novae, X-ray bursts). The code solves the standard set of differential equations of stellar evolution: conservation of mass, momentum, and energy, and energy transport by radiation and time-dependent convection. The equation of state includes contributions from the degenerate electron gas, the ion plasma, and radiation. Coulomb corrections to the electron pressure are taken into account. Radiative and conductive opacities are considered. The code is linked to a nuclear reaction network that contains $\sim 120$ nuclear species, from $\mathrm{H}$ to ${ }^{48} \mathrm{Ti}$, connected through $630 \mathrm{nu}-$ clear interactions, with updated STARLIB rates (Sallaska et al. 2013). Additional details on the SHIVA code can be found in José \& Hernanz (1998) and José (2016).

The nova models computed in this work assume a $\sim 1.0 M_{\odot}$ CO white dwarf, resulting from the evolution of an $8 M_{\odot}$ progenitor star, self-consistently computed throughout the successive $\mathrm{H}$ - and He-burning stages with the HYADES code (see Halabi et al. 2012, and references therein). The database of the $\mathrm{Nu}-$ clear Astrophysics Compilation of Reaction Rates (hereafter, NACRE; Angulo et al. 1999) was used, in particular for the triple- $\alpha$ and ${ }^{12} \mathrm{C}(\alpha, \gamma)$ reactions. The chemical structure of the resulting CO-rich white dwarf is shown in Fig. 1. Qualitatively similar chemical profiles have been obtained for the remnants of $6 M_{\odot}$ and $7 M_{\odot}$ progenitor stars.

Most of the simulations of CO novae (i.e., non-neon novae) performed to date have traditionally assumed that the outer

\footnotetext{
2 See Gehrz et al. (1998), Gehrz (2008), and José \& Shore (2008) for examples of $\mathrm{O}>\mathrm{C}$ ejecta observed in $\mathrm{CO}$ novae at different stages of the outburst.
}

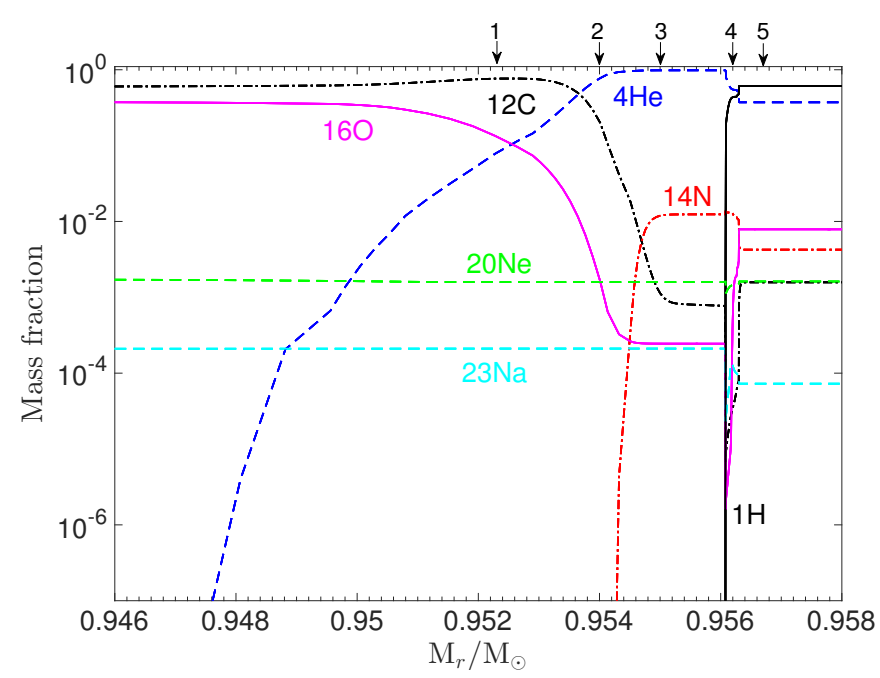

Fig. 1. Chemical profiles after a series of thermal pulses following completion of central He-burning for an $8 M_{\odot}$ star, computed with the HYADES code.

layers of the white dwarf hosting the outburst have equal mass fractions of ${ }^{12} \mathrm{C}$ and ${ }^{16} \mathrm{O}$ (some include a marginal amount of leftover ${ }^{22} \mathrm{Ne}$, at a level of $1 \%$ by mass, but maintaining $\left.X\left({ }^{12} \mathrm{C}\right) / X\left({ }^{16} \mathrm{O}\right)=1\right)$. However, a closeup look at Fig. 1 reveals instead a chemical abundance pattern dominated by the presence of ${ }^{12} \mathrm{C}$ over ${ }^{16} \mathrm{O}$ in the outer white dwarf layers (with the exception of the outermost, unburnt H-rich layers).

In the simulations discussed in Sect. 3, the white dwarf is assumed to accrete solar composition material $(Z \sim 0.02)$ from a low-mass stellar companion at a characteristic mass-accretion rate of $\dot{M} \sim 2 \times 10^{-10} M_{\odot} \mathrm{yr}^{-1}$. The accreted material is mixed with material dredged up from the underlying white dwarf (a mixture with $75 \%$ solar composition plus $25 \%$ outer white dwarf material has been adopted in all the calculations reported in this paper; see Kelly et al. 2013, for a recent reanalysis of mixing fractions in novae supporting a $25 \%$ white dwarf contribution; see also Casanova et al. 2010, 2011a,b, for 2D and 3D hydrodynamic simulations of mixing at the core-envelope interface during nova outbursts).

To specifically investigate the role played by the composition of the underlying white dwarf, six different nova models have been evolved. Models 1 to 5 are identical, except for the composition of the substrate with which the solar-like accreted material mixes. To this end, five different locations in the outer white dwarf layers have been considered (see points indicated by arrows in Fig. 1). For comparison, an additional model (Model 6) has been evolved under the traditional assumption of equal mass fractions of ${ }^{12} \mathrm{C}$ and ${ }^{16} \mathrm{O}$ in the outer white dwarfs layers.

\section{Results}

\subsection{Model 1}

The importance of the initial $\mathrm{C} / \mathrm{O}$ ratio in the outer layers of the underlying white dwarf, and specifically, the initial ${ }^{12} \mathrm{C}$ mass fraction in the envelope is described in the framework of the evolution of Model 1. In this particular model, the solar material transferred from the secondary is assumed to mix with a C-rich substrate whose composition is indicated in the upper part of Fig. 1 as point 1 (see also Table 1). The early accretion phase is dominated both by the pp chains (mainly through $\left.{ }^{1} \mathrm{H}\left(\mathrm{p}, \mathrm{e}^{+} v\right)^{2} \mathrm{H}\right)$ and by the $\mathrm{CNO}$-cycle reaction ${ }^{12} \mathrm{C}(\mathrm{p}, \gamma){ }^{13} \mathrm{~N}$, 
Table 1. Initial composition of the accreted envelopes.

\begin{tabular}{ccccc}
\hline \hline Model & ${ }^{1} \mathrm{H}$ & ${ }^{12} \mathrm{C}$ & ${ }^{16} \mathrm{O}$ & $\mathrm{CNO}$ \\
\hline 1 & 0.529 & 0.193 & $4.11-2$ & 0.235 \\
2 & 0.529 & $5.21-2$ & $7.95-3$ & $6.10-2$ \\
3 & 0.529 & $2.49-3$ & $7.61-3$ & $1.42-2$ \\
4 & 0.638 & $2.28-3$ & $7.76-3$ & $1.40-2$ \\
5 & 0.682 & $2.67-3$ & $9.51-3$ & $1.42-2$ \\
6 & 0.529 & 0.126 & 0.131 & 0.258 \\
\hline
\end{tabular}

followed by ${ }^{13} \mathrm{~N}\left(\beta^{+}\right){ }^{13} \mathrm{C}$. When the temperature at the envelope base reaches $T_{\text {base }}=2.4 \times 10^{7} \mathrm{~K}$, the nuclear timescale becomes shorter than the accretion timescale, and accretion is no longer relevant. The rate of nuclear energy generation reaches $\sim 10^{8} \mathrm{erg} \mathrm{g}^{-1} \mathrm{~s}^{-1}$ at the innermost envelope shell. The total mass piled on top of the white dwarf at the end of this accretion stage (which lasts $\sim 1.7 \times 10^{5} \mathrm{yr}$ ) is $\sim 3.9 \times 10^{-5} M_{\odot}$ (see Table 2 , for details).

Shortly afterwards, degeneracy is lifted at the envelope base, so that pressure becomes sensitive to temperature. Consequently, the envelope begins to expand after achieving a maximum pressure of $P_{\text {max,base }}=8.6 \times 10^{18} \mathrm{dyn} \mathrm{cm}^{-2}$ and a maximum density of $\rho_{\text {max,base }}=3.6 \times 10^{3} \mathrm{~g} \mathrm{~cm}^{-3}$. The beginning of the dynamic stage (i.e., a thermonuclear runaway) is accompanied by the development of superadiabatic gradients at the innermost layers of the envelope. This spawns the onset of convection, which progressively expands throughout the envelope. At $T_{\text {base }}=5 \times 10^{7} \mathrm{~K}$, the nuclear activity is fully dominated by the cold CNO cycle, mainly through ${ }^{12} \mathrm{C}(\mathrm{p}, \gamma){ }^{13} \mathrm{~N}\left(\beta^{+}\right){ }^{13} \mathrm{C}(\mathrm{p}, \gamma){ }^{14} \mathrm{~N}$, while no significant activity in the $\mathrm{NeNa}$ and $\mathrm{MgAl}$-mass region is noticed. A similar behavior is found at $10^{8} \mathrm{~K}$, when convection has already extended throughout the entire envelope, with a characteristic turnover time of about $1 \mathrm{~s}$. However, the dominant ${ }^{12} \mathrm{C}(\mathrm{p}, \gamma){ }^{13} \mathrm{~N}\left(\beta^{+}\right){ }^{13} \mathrm{C}(\mathrm{p}, \gamma){ }^{14} \mathrm{~N}$ reactions are now significantly supplemented by ${ }^{13} \mathrm{~N}(\mathrm{p}, \gamma){ }^{14} \mathrm{O}\left(\beta^{+}\right){ }^{14} \mathrm{~N}(\mathrm{p}, \gamma){ }^{15} \mathrm{O}$ and ${ }^{16} \mathrm{O}(\mathrm{p}, \gamma){ }^{17} \mathrm{~F}$; there is still a marginal contribution from reactions of the NeNa and $\mathrm{MgAl}$ mass-regions (mainly, ${ }^{24} \mathrm{Mg}(\mathrm{p}, \gamma){ }^{25} \mathrm{Al}$ ). The star has spent about $1.2 \times 10^{6} \mathrm{~s}$ raising its temperature at the innermost envelope layers from $3 \times 10^{7} \mathrm{~K}$ to $10^{8} \mathrm{~K}$.

About $100 \mathrm{~s}$ later, when the temperature reaches $T_{\text {base }}=$ $1.66 \times 10^{8} \mathrm{~K}$, the star achieves a maximum rate of nuclear energy generation of $3 \times 10^{16} \mathrm{ergs}^{-1} \mathrm{~s}^{-1}$. Forty seconds later, the envelope base achieves a peak temperature of $T_{\text {base,max }}=1.72 \times 10^{8} \mathrm{~K}$. At this stage, nuclear energy is mostly released through the hot $\mathrm{CNO}$ cycle reactions (HCNO1) $\left.{ }^{13} \mathrm{~N}(\mathrm{p}, \gamma){ }^{14} \mathrm{O}\left(\beta^{+}\right)\right)^{14} \mathrm{~N}$, supplemented by ${ }^{12} \mathrm{C}(\mathrm{p}, \gamma){ }^{13} \mathrm{~N}\left(\beta^{+}\right){ }^{13} \mathrm{C}(\mathrm{p}$, $\gamma){ }^{14} \mathrm{~N}(\mathrm{p}, \gamma){ }^{15} \mathrm{O}\left(\beta^{+}\right){ }^{15} \mathrm{~N}(\mathrm{p}, \alpha){ }^{12} \mathrm{C}$ and ${ }^{16} \mathrm{O}(\mathrm{p}, \gamma){ }^{17} \mathrm{~F}\left(\beta^{+}\right){ }^{17} \mathrm{O}(\mathrm{p}$, $\alpha)^{14} \mathrm{~N}$. Secondary nuclear activity involves a series of protoncapture reactions and $\beta^{+}$decays of a handful of species in the $\mathrm{NeNa}-\mathrm{MgAl}$ mass regions (e.g., ${ }^{23} \mathrm{Na},{ }^{24,25,26} \mathrm{Mg},{ }^{25} \mathrm{Al}$, and both the ground and first isomeric states of $\left.{ }^{26} \mathrm{Al}\right)$. A small leakage from the $\mathrm{MgAl}$-mass region is driven by proton captures on ${ }^{27} \mathrm{Si}$ and ${ }^{27} \mathrm{Al}$.

A fraction of the nuclear energy released during the event is transformed into kinetic energy, which powers the ejection of $3.2 \times 10^{-5} M_{\odot}$ of nuclear-processed material, with a mean velocity of $1540 \mathrm{~km} \mathrm{~s}^{-1}$. As confirmed by the large concentrations of hydrogen and carbon in the ejecta (see Table 3), the runaway is halted by envelope expansion rather than by fuel consumption.

\subsection{Models 2 to 6}

As shown by Shara (1981) and Fujimoto (1982), the key parameter that determines the strength of a nova outburst is the pressure achieved at the core-envelope interface, $P_{\text {base }}$, a measure of the overall pressure exerted by the layers overlying the ignition shell:

$P_{\text {base }}=\frac{G M_{\mathrm{wd}}}{4 \pi R_{\mathrm{wd}}^{4}} M_{\mathrm{acc}}$.

In Eq. (1), $M_{\mathrm{wd}}$ and $R_{\mathrm{wd}}$ are the mass and radius of the white dwarf hosting the eplosion, and $M_{\text {acc }}$ is the mass of the accreted envelope. To drive mass ejection, pressures in the range $P_{\text {base }} \sim$ $10^{19}-10^{20} \mathrm{dyn}^{-2}$ are required at the base of the envelope, the exact value depending on the chemical composition (Fujimoto 1982; MacDonald 1983). For a given pressure, Eq. (1) reveals that the mass of the accreted envelope depends only on the mass of the underlying white dwarf star because of the relationship between stellar mass and radius. As shown in Table 2, the larger the mass of the accreted envelope, the larger the pressure at its base. This translates into a more violent outburst characterized by a larger peak temperature, $T_{\text {base,max }}$ (Table 2 ).

The analysis reported for Model 1 reveals that the single most important reaction during the early stages of a nova outburst ${ }^{3}$ is ${ }^{12} \mathrm{C}(\mathrm{p}, \gamma)$. The rate at which these two species interact per second and per unit volume can, in general, be expressed as (see, e.g., Iliadis 2015, for details)

$r_{\mathrm{HC}}=n_{\mathrm{H}} n_{\mathrm{C}}\langle\sigma v\rangle_{\mathrm{HC}}$,

where $n_{\mathrm{H}}$ and $n_{\mathrm{C}}$ denote the number densities of ${ }^{1} \mathrm{H}$ and ${ }^{12} \mathrm{C}$ in the stellar plasma, respectively, and $\langle\sigma v\rangle_{\mathrm{HC}}$ is the reaction rate per particle pair. Equation (2) reveals that the rate of interaction is proportional to the mass fractions of the interacting species. Therefore, the most relevant quantity to determine the strength of the outburst is not the amount of ${ }^{12} \mathrm{C}$, as is frequently stated, but the product $\left[X\left({ }^{1} \mathrm{H}\right) \times X\left({ }^{12} \mathrm{C}\right)\right]_{\text {ini }}$ at the beginning of the accretion stage. A reduction in this quantity delays ignition, since fewer nuclear reactions occur and, hence, less energy is released. This translates into a rise in the characteristic timescale for accretion, which subsequently leads to a larger accreted mass, larger pressure at the base, and in the end, a more violent outburst ${ }^{4}$. Indeed, in the models reported in this work, a reduction in $\left[X\left({ }^{1} \mathrm{H}\right) \times\right.$ $\left.X\left({ }^{12} \mathrm{C}\right)\right]_{\text {ini }}$ by a factor of $\sim 80$ (see, e.g., Models 1 and 3 in Table 2 ) translates into an increase by a factor $\sim 2$ in the maximum pressure attained at the base of the envelope, and in the amount of mass accreted and ejected (with a smaller effect on the peak temperature achieved in the outburst as well).

\subsection{Nucleosynthesis and implications for dust formation}

The final, mass-averaged composition of the ejecta accompanying the set of nova outbursts calculated in this work is summarized for CNO-group species in Table 3.

In Model 1 , the ejecta is dominated by high concentrations of $\mathrm{H}(0.498$, by mass $),{ }^{4} \mathrm{He}(0.240)$, and ${ }^{14} \mathrm{~N}(0.106)$. This is followed by ${ }^{13} \mathrm{C},{ }^{16} \mathrm{O},{ }^{12} \mathrm{C}$, and other $\mathrm{CNO}$-group nuclei. A similar pattern is obtained in Models 2 to 5 , except that both ${ }^{12,13} \mathrm{C}$ are individually more abundant than any of the stable $\mathrm{O}$ isotopes, ${ }^{16,17,18} \mathrm{O}$. It is worth noting that Models 1 to 5 yield C-rich ejecta.

\footnotetext{
3 Specifically for CO novae. See Shen \& Bildsten (2009) for ignition conditions in C-poor envelopes.

4 See José et al. (2007) for a study of nova outbursts in metal-poor, primordial-like binaries.
} 
Table 2. Main characteristics of the nova outbursts.

\begin{tabular}{cccccccc}
\hline \hline Model & {$\left[X\left({ }^{12} \mathrm{C}\right) \times X\left({ }^{1} \mathrm{H}\right)\right]_{\text {ini }}$} & $\begin{array}{c}P_{\text {base,max }} \\
\left.\left(10^{19} \mathrm{dyn} \mathrm{cm}\right)^{-2}\right)\end{array}$ & $\begin{array}{c}M_{\text {acc }} \\
\left(10^{-5} M_{\odot}\right)\end{array}$ & $\begin{array}{c}T_{\text {base,max }} \\
\left(10^{8} \mathrm{~K}\right)\end{array}$ & $\begin{array}{c}M_{\text {ejec }} \\
\left(10^{-5} M_{\odot}\right)\end{array}$ & $\begin{array}{c}V_{\text {mean }} \\
\left(\mathrm{km} \mathrm{s}^{-1}\right)\end{array}$ & $\begin{array}{c}K_{\text {mean }} \\
\left(10^{44} \mathrm{erg}\right)\end{array}$ \\
\hline 1 & 0.102 & 0.857 & 3.94 & 1.72 & 3.19 & 1540 & 9.25 \\
2 & $2.76-2$ & 1.09 & 5.05 & 1.78 & 4.08 & 1210 & 7.26 \\
3 & $1.32-3$ & 1.52 & 7.07 & 1.89 & 5.71 & 1060 & 8.18 \\
4 & $1.46-3$ & 1.43 & 6.72 & 1.83 & 5.43 & 983 & 6.81 \\
5 & $1.82-3$ & 1.37 & 6.43 & 1.80 & 5.20 & 954 & 6.19 \\
6 & $6.67-2$ & 0.901 & 4.15 & 1.73 & 3.35 & 1400 & 7.90 \\
\hline
\end{tabular}

Table 3. Mean composition of the ejecta (CNO-group nuclei).

\begin{tabular}{ccccccccc}
\hline \hline Model & ${ }^{12} \mathrm{C}$ & ${ }^{13} \mathrm{C}$ & ${ }^{14} \mathrm{~N}$ & ${ }^{15} \mathrm{~N}$ & ${ }^{16} \mathrm{O}$ & ${ }^{17} \mathrm{O}$ & ${ }^{18} \mathrm{O}$ & Ejecta \\
\hline 1 & $2.90-2$ & $7.56-2$ & $1.06-1$ & $8.42-3$ & $3.85-2$ & $8.36-4$ & $5.27-7$ & C-rich \\
2 & $8.89-3$ & $1.43-2$ & $3.56-2$ & $2.73-3$ & $5.62-3$ & $1.46-4$ & $9.23-8$ & C-rich \\
3 & $2.04-3$ & $3.02-3$ & $7.21-3$ & $5.26-4$ & $3.07-4$ & $9.46-6$ & $6.52-9$ & C-rich \\
4 & $1.79-3$ & $2.66-3$ & $7.73-3$ & $4.04-4$ & $3.92-4$ & $1.01-5$ & $7.19-9$ & C-rich \\
5 & $1.63-3$ & $2.47-3$ & $8.08-3$ & $3.44-4$ & $5.09-4$ & $1.14-5$ & $8.24-9$ & C-rich \\
6 & $2.05-2$ & $4.17-2$ & $8.20-2$ & $7.26-3$ & $1.17-1$ & $2.75-3$ & $1.72-6$ & O-rich \\
\hline
\end{tabular}

Model 6, however, presents an important deviation: while $\mathrm{H}$ and ${ }^{4} \mathrm{He}$ still present the largest mass fractions in the ejecta, the third most abundant species is now, by far, ${ }^{16} \mathrm{O}$. In fact, the large concentration of this isotope $\left(X\left({ }^{16} \mathrm{O}\right)=0.117\right.$, by mass $)$ results in O-rich ejecta.

While the use of state-of-the-art chemical profiles for COrich white dwarfs hosting nova outbursts has a limited impact on the dynamics of the nova outburst (i.e., mass ejected, peak temperature, nucleoynthesis), it has a dramatic effect on the properties of the dust expected to condense in those environments. To date, $\mathrm{CO}$ novae have been traditionally ruled out as likely progenitors of $\mathrm{C}$-rich grains, on the basis of the O-rich ejecta obtained when equal mass fractions of ${ }^{12} \mathrm{C}$ and ${ }^{16} \mathrm{O}$ are adopted (see, e.g., José et al. 2004). In sharp contrast, the models reported in this paper show, however, that the implementation of realistic chemical profiles leads to C-rich ejecta, instead. This opens up new possibilities for the contribution of novae to the inventory of presolar grains identified in meteorites, particularly in a number of carbonaceous phases (i.e., nanodiamonds, silicon carbides, and graphites).

\section{Discussion}

As shown in Sect. 3, the use of state-of-the-art chemical profiles for the white dwarf that hosts a $\mathrm{CO}$ nova outburst turns out to be critical for the production of C-rich ejecta. Even though realistic white dwarf models with similar profiles have been reported in the literature (see, e.g., Salaris et al. 1997; Karakas \& Lugaro 2016), it is worth analyzing the possible role played by nuclear uncertainties in this regard, both during the previous stages of the evolution of the progenitor and during the nova outburst itself.

\subsection{Uncertainties in nova nucleosynthesis}

A significant fraction of the nuclear reactions of interest for nova nucleosynthesis that involve stable nuclei (mostly protoninduced reactions) have already been measured directly in the laboratory (José et al. 2006).

For the case of $\mathrm{CO}$ novae, in which the main nuclear path does not extend significantly beyond the CNOF mass region, current reaction rate uncertainties (i.e., $\leq 30 \%$ ) do not dramatically affect model predictions. Indeed, after a number of improvements on the ${ }^{17} \mathrm{O}(\mathrm{p}, \alpha)$ and ${ }^{17} \mathrm{O}(\mathrm{p}, \gamma)$ rates, the main source of uncertainty in this region is mostly dominated by the challenging reaction ${ }^{18} \mathrm{~F}(\mathrm{p}, \alpha)$, which - under explosive conditions drives the main nuclear path down to ${ }^{15} \mathrm{O}$ in both hot CNO cycles 2 and 3 (see, e.g., Iliadis 2015; José 2016). It is, however, worth noting that the uncertainty associated with ${ }^{18} \mathrm{~F}(\mathrm{p}, \alpha)$ has an impact on the expected $\mathrm{O}$ yields for ONe novae, but not for CO novae (Iliadis et al. 2002).

All in all, we conclude that the $\mathrm{CO}$ nova nucleosynthesis predictions reported in this work are not affected by current reaction-rate uncertainties.

\subsection{Uncertainties in the evolution of the progenitor star}

The evolution of a star throughout the $\mathrm{H}$ - and He-burning stages up to the formation of a CO-rich core may be affected by uncertainties in two key nuclear reaction rates.

The first is the triple-alpha reaction (hereafter, $3 \alpha$ ). Models presented in this work rely on chemical profiles for the white dwarf based on stellar evolution computed with the HYADES code, for which the NACRE $3 \alpha$ rate was adopted (Angulo et al. 1999). Since the NACRE compilation, several prescriptions for this rate have been published. Through a measurement of the inverse process, ${ }^{12} \mathrm{C} \rightarrow 3{ }^{4} \mathrm{He}$, Fynbo et al. (2005) determined a new $3 \alpha$ rate for temperatures ranging between $10^{7} \mathrm{~K}$ and $10^{10} \mathrm{~K}$. The new rate was found to be higher than the NACRE rate for temperatures below $\sim 5 \times 10^{7} \mathrm{~K}$, while much smaller than NACRE above $10^{9} \mathrm{~K}$. The potential effect of the Fynbo et al. $3 \alpha$ rate has been checked by means of new evolutionary sequences of an $8 M_{\odot}$ star computed with the HYADES code throughout the He-burning stage. Oxygen becomes higher than carbon in the inner regions of the star, but as in the chemical profiles adopted in this work, the outer layers still remain C-rich. More recently, Ogata et al. (2009) have reported on a new rate based on quantum mechanical calculations of the three-body Schrödinger equation. Around $10^{7} \mathrm{~K}$, the rate exhibited an increase of 20 orders of magnitude over the standard NACRE rate. It is worth noting, however, that this rate has been refuted by more recent 
calculations, and differences with respect to NACRE at low temperatures are now reduced to less than an order of magnitude (see, e.g., Ishikawa 2013; Akahori et al. 2015, for details).

The second reaction is ${ }^{12} \mathrm{C}(\alpha, \gamma){ }^{16} \mathrm{O}$. It is a key reaction determining the final $\mathrm{C} / \mathrm{O}$ ratio wherever He-burning takes place, and dominates over the triple- $\alpha$ reaction when the helium mass fraction drops below about 0.1 . The rate of this reaction is dominated by several subthreshold resonances and still remains uncertain, even though it has been re-evaluated several times since NACRE (e.g., Katsuma et al. 2012; Xu et al. 2013). The Katsuma et al. rate is higher than the NACRE rate for temperatures above $\geq 3 \times 10^{8} \mathrm{~K}$, while the $\mathrm{Xu}$ et al. rate exceeds the NACRE rate above $\geq 5 \times 10^{8} \mathrm{~K}$. To estimate the potential effect of the Heburning rates on the results reported in this paper, new evolutionary sequences have been computed with the HYADES code using the Fynbo et al. and the $\mathrm{Xu}$ et al. prescriptions for the $3 \alpha$ and ${ }^{12} \mathrm{C}(\alpha, \gamma){ }^{16} \mathrm{O}$ rates, respectively. The implementation of these rates results in an important increase in the oxygen abundance in the inner layers of the star (up to $\sim 0.75 M_{\odot}$ at the end of Heburning); however, the outer layers are still C-rich.

To investigate how our conclusions are affected by the latest He-burning rate determinations, we recomputed an $8 M_{\odot}$ model using the Fynbo et al. (2005) rate for the $3 \alpha$ reaction and $\mathrm{Xu}$ et al. (2013) for the ${ }^{12} \mathrm{C}(\alpha, \gamma)$ reaction. We found that the outer core remains C-rich. A $6 M_{\odot}$ model by Karakas \& Lugaro (2016) using the Fynbo et al. (2005) rate and the Xu et al. (2013) rate for the $3 \alpha$ and the ${ }^{12} \mathrm{C}+\alpha$ reactions, respectively, yields similar conclusions.

A very recent work by Fields et al. (2016), available as a preprint during completion of this manuscript, analyzes the structural and compositional properties of $\mathrm{CO}$ white dwarfs in connection with uncertainties in the $\mathrm{H}$ - and He-burning rates obtained by a new Monte Carlo sampling procedure. The $\mathrm{CO}$ white dwarf remnant of their $3 M_{\odot}$ star exhibits a C-rich outer core, which independently shows that our nova nucleosynthesis predictions apply to lower progenitor masses. By evolving a grid of evolutionary models of 1 to $6 M_{\odot}$, they find that rate uncertainties, especially those affecting the $3 \alpha$ and ${ }^{12} \mathrm{C}(\alpha, \gamma)$ reactions, do not have a large impact on the final white dwarf mass distribution, but can significantly affect the central ${ }^{12} \mathrm{C}$ and ${ }^{16} \mathrm{O}$ abundances. Since uncertainty factors are likely temperaturedependant, it is unclear how the $\mathrm{C}$ and $\mathrm{O}$ profiles in the outer core would be affected, which is the region of interest for nova nucleosynthesis. As a conclusion, current nuclear uncertainties do not significantly affect the formation of C-rich layers in the outer part of $\mathrm{CO}$ white dwarfs, and hence, the production of $\mathrm{C}$-rich ejecta in $\mathrm{CO}$ nova outbursts.

It is finally worth noting that production of C-rich ejecta in nova outbursts may also account for the origin of C-rich J-type stars. These stars represent $10 \%-15 \%$ of the observed $\mathrm{C}$ stars in our Galaxy and in the Large Magellanic Cloud. Indeed, a binarystar formation channel for C-rich J-type stars has been recently proposed in the framework of re-accretion of $\mathrm{C}$-rich nova ejecta onto main sequence companions (Sengupta et al. 2013).

Acknowledgements. We thank the referee, Robert Gehrz, for very positive feedback on the manuscript. J.J. would like to express his gratitude to the Center of Advanced Mathematical Sciences, American University of Beirut (AUB, Lebanon), for financial support and hospitality during his visit to AUB, where part of this project started. This work has been partially supported by the Spanish MINECO grant AYA2014-59084-P, by the E.U. FEDER funds, and by the AGAUR/Generalitat de Catalunya grant SGR0038/2014 (J.J.). G.H. thanks
Christopher Tout and Robert Izzard for helpful discussions, the STFC for funding her postdoctoral research at the Institute of Astronomy, and the FAS at the American University of Beirut where part of the calculations were performed.

\section{References}

Akahori, T., Funaki, Y., \& Yabana, K. 2015, Phys. Rev. C, 92, 022801

Amari, S. 2002, New Astron. Rev., 46, 519

Amari, S., Gao, X., Nittler, L. R., et al. 2001, ApJ, 551, 1065

Andersen, A. C. 2011, in Why Galaxies Care about AGB Stars II: Shining Examples and Common Inhabitants, eds. F. Kerschbaum, T. Lebzelter, \& R. F. Wing (San Francisco: ASP Conf. Ser.), 215

Angulo, C., Arnould, M., Rayet, M., et al. 1999, Nucl. Phys. A, 656, 3

Casanova, J., José, J., García-Berro, E., Calder, A., \& Shore, S. N. 2010, A\&A, 513, L5

Casanova, J., José, J., García-Berro, E., Calder, A., \& S. N. Shore 2011a, A\&A, 527, A5

Casanova, J., José, J., García-Berro, E., Shore, S. N., \& Calder, A. C. 2011b, Nature, 478, 490

Clayton, D. D., \& Hoyle, F. 1976, ApJ, 203, 490

Evans, A. 1990, in Physics of Classical Novae, eds. A. Cassatella, \& R. Viotti (Berlin: Springer-Verlag), 253

Evans, A., \& Rawlings, M. C. 2008, in Classical Novae, eds. M. F. Bode, \& A. Evans, 2nd edn. (Cambridge, UK: Cambridge University Press), 308

Fields, C. E., Farmer, R., Petermann, I., Iliadis, C., \& Timmes, F. X. 2016, ApJ, 823,46

Fujimoto, M. Y. 1982, ApJ, 257, 752

Fynbo, H. O. U., Diget, C. A., Bergmann, U. C., et al. 2005, Nature, 433, 136

Gehrz, R. D. 1990, in Physics of Classical Novae, eds. A. Cassatella, \& R. Viotti (Berlin: Springer-Verlag), 138

Gehrz, R. D. 2002, in Classical Nova Explosions, eds. M. Hernanz, \& J. José (Melville: American Inst. Phys.), 198

Gehrz, R. D. 2008, in Classical Novae (2nd Ed.), eds. M. F. Bode, \& A. Evans (Cambridge, UK: Cambridge University Press), 167

Gehrz, R. D., Truran, J. W., Williams, R. E., \& Starrfield, S. 1998, PASP, 110, 3

Haenecour, P., Floss, C., José, J., et al. 2016, ApJ, 825, 88

Halabi, G. M., El Eid, M. F., \& Champagne, A. 2012, ApJ, 761, 10

Iliadis, C. 2015, Nuclear Physics of Stars, 2nd edn. (Weinheim: Wiley-VCH Verlag)

Iliadis, C., Champagne, A., José, J., Starrfield, S., \& Tupper, P. 2002, ApJS, 142, 105

Ishikawa, S. 2013, Phys. Rev. C, 87, 055804

José, J. 2016, Stellar Explosions: Hydrodynamics and Nucleosynthesis (Boca Raton: CRC/Taylor and Francis)

José, J., \& Hernanz, M. 1998, ApJ, 494, 680

José, J., \& Shore, S. N. 2008, in Classical Novae, eds. M. F. Bode, \& A. Evans, 2nd edn. (Cambridge, UK: Cambridge University Press), 121

José, J., Hernanz, M., Amari, S., Lodders, K., \& Zinner, E. 2004, ApJ, 612, 414 José, J., Hernanz, M., \& Iliadis, C. 2006, Nucl. Phys. A, 777, 550

José, J., García-Berro, E., Hernanz, M., \& Gil-Pons, P. 2007, ApJ, 662, L103

Karakas, A., \& Lugaro, M. 2016, ApJ, submitted

Katsuma, M. 2012, ApJ, 745, 192

Kelly, K. J., Iliadis, C., Downen, L., José, J., \& Champagne, A. 2013, ApJ, 777, 130

Kovetz, A., \& Prialnik, D. 1997, ApJ, 477, 356

Lodders, K. 2003, ApJ, 591, 1220

MacDonald, J. 1983, ApJ, 267, 732

Ogata, K., Kan, M., \& Kamimura, M. 2009, Progr. Theor. Phys., 122, 1055

Pepin, R. O., Palma, R. L., Gehrz, R. D., \& Starrfield, S. 2011, ApJ, 742, 86

Sallaska, A. L., Iliadis, C., Champange, A. E., et al. 2013, ApJS, 207, 18

Sedlmayr, E. 1994, in Molecules in the Stellar Environment, ed. U. G. Jorgensen

(Berlin: Springer-Verlag), 163

Sengupta, S., Izzard, R. G., \& Lau, H. H. B. 2013, A\&A, 559, A66

Shara, M. M. 1981, ApJ, 243, 926

Shen, K., \& Bildsten, L. 2009, ApJ, 692, 324

Shore, S. N., \& Gehrz, R. D. 2004, A\&A, 417, 695

Shore, S. N., Starrfield, S., Gonzalez-Riestra, R., Hauschildt, P. H., \& Sonneborn, G. 1994, Nature, 369, 539

Stratton, F. J. M., \& Manning, W. H. 1939, Atlas of Spectra of Nova Herculis 1934 (Cambridge, UK: Solar Physics Observ.)

Xu, Y., Takahashi, K., Goriely, S., et al. 2013, Nucl. Phys. A, 918, 61 